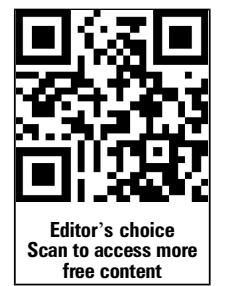

${ }^{1}$ Risk Factor Monitoring and Methods Branch, Applied Research Program, National Cancer Institute, Bethesda, Maryland, USA

${ }^{2}$ Diabetes, Endocrinology, and Obesity Branch, Intramural Research Program, National Institute of Diabetes and Digestive and Kidney Diseases, Bethesda, Maryland, USA

Correspondence to Dr Richard P Troiano, Risk Factor Monitoring and Methods Branch, Applied Research Program, National Cancer Institute, $\mathrm{NCl}$ Shady Grove, 9609 Medical Center Dr, MSC 9762, Bethesda, MD 20892-9762, USA; troianor@mail.nih.gov

Accepted 2 April 2014 Published Online First 29 April 2014

\title{
Evolution of accelerometer methods for physical activity research
}

\author{
Richard P Troiano, ${ }^{1}$ James J McClain, ${ }^{1}$ Robert J Brychta, ${ }^{2}$ Kong Y Chen ${ }^{2}$
}

\section{ABSTRACT}

The technology and application of current accelerometerbased devices in physical activity (PA) research allow the capture and storage or transmission of large volumes of raw acceleration signal data. These rich data not only provide opportunities to improve PA characterisation, but also bring logistical and analytic challenges. We discuss how researchers and developers from multiple disciplines are responding to the analytic challenges and how advances in data storage, transmission and big data computing will minimise logistical challenges. These new approaches also bring the need for several paradigm shifts for PA researchers, including a shift from count-based approaches and regression calibrations for PA energy expenditure (PAEE) estimation to activity characterisation and EE estimation based on features extracted from raw acceleration signals. Furthermore, a collaborative approach towards analytic methods is proposed to facilitate PA research, which requires a shift away from multiple independent calibration studies. Finally, we make the case for a distinction between PA represented by accelerometer-based devices and PA assessed by self-report.

Accelerometer-based devices are now commonly used to characterise physical activity (PA) behaviour in research and consumer applications. In this article, we present several topics related to the growth in use of accelerometers that should be of interest to PA researchers and research consumers. A review of the history of accelerometers in PA research provides the background. This is followed by a discussion of new approaches to analyse acceleration data that are feasible because of technological advances in devices and computing. Two related projects of interest to the PA research community are described: a collaborative effort to harmonise accelerometer data analysis and the collection of accelerometer data in the US National Health and Nutrition Examination Survey (NHANES) 2011-2014. The article concludes with further observations based on our experience with accelerometer-based devices in population monitoring.

\section{BRIEF HISTORY OF ACCELEROMETER-BASED PA RESEARCH}

The first accelerometer-based PA monitor to be adopted by researchers was developed in the $1980 \mathrm{~s},{ }^{1}$ and the 1990 s saw a proliferation of new research devices. During these early years, accelerometer-based devices were seen as an intriguing, although niche, assessment technology due to limitations that included high device cost, as well as reliability, calibration and validity concerns. ${ }^{2}$ While many of these challenges are still being debated today, by the early 2000s, accelerometer technology was more accessible and the apparent value of objective PA data collected over multiple free-living days had grown attractive to many PA researchers. ${ }^{3}$ The application of accelerometers as a measure of PA has expanded exponentially. In 2004, an examination of the number of published articles mentioning PA or exercise and accelerometer or accelerometry found that the publication rate had increased from roughly 10 or fewer per year in 1981-1996 to nearly 90 per year in 2003 and $2004 .^{3} \mathrm{~A}$ recent update of that publication count finds that more than 600 articles per year were published in 2012 and 2013 (figure 1).

The 2004 publication count was conducted for a conference titled 'Objective measurement of physical activity: closing the gaps in the science of accelerometry.' Presentations at the workshop, which were summarised in a supplement to Medicine and Science in Sports and Exercise (Med Sci Sports Exerc 2005 Nov;37(11 Suppl)), highlighted the developments of the technologies and critical needs of the research community. The desires included accurate assessments of certain activity types, improved energy expenditure (EE) estimation and statistical models and unified calibration and validation studies. One major limitation of the accelerometry technologies at the time was minute-by-minute time resolution and 'counts' as the standard output. A critical mass of behavioural and clinical researchers and engineers at the conference made a call to manufacturers of accelerometer-based devices to open up their proprietary data processing algorithms so that (count) data from different manufacturers' devices could be more transparent and easily compared. ${ }^{2}$

The technical advances in accelerometry sensors and other microelectronics since the 2004 conference are nothing short of remarkable. A portable accelerometer-based PA monitor can now be built with much larger memory and battery capacities, wider acceleration range, better linearity, smaller size and, in most cases, for a lower cost than several years ago. At the same time, the potential benefits for researchers to have access to the raw acceleration signal data, rather than the manufacturer-specific 'counts' data, have also been realised. ${ }^{4}$ Further, increased utilisation of accelerometer technology within national surveillance and prospective cohorts has driven interest in archiving data to support the ability to reprocess raw acceleration signals based on evolving analytic methods over a period of years or decades. Motivated by these developments, manufacturers have responded by providing devices that capture and store raw acceleration signals at sampling frequencies up to $100 \mathrm{~Hz}$. 
Figure 1 Publications by year with search terms 'exercise or physical activity' and 'accelerometer or accelerometry,' Scopus.com, accessed 3 January 2014.

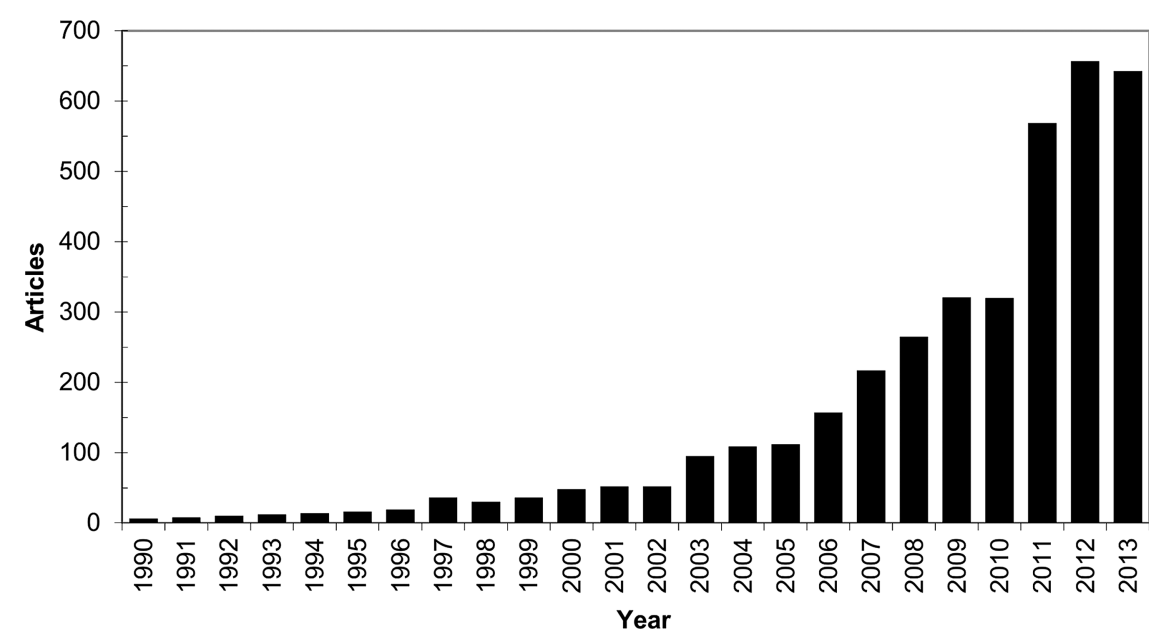

Despite the technical advances in the monitor hardware, many current study outcomes are still based on earlier analytic approaches, such as linear regressions for predicting EE and regression-based cut-points to classify time spent in different activity intensities. Use of these analytic approaches is understandable because researchers continue to use many count-based accelerometers from past studies for economic reasons or to maintain comparability with earlier data collections. Not surprisingly, conflicts and debates about which monitor(s) to use, where to position them on the body, and how to process the data are still common in the field of PA research. However, the path forward needs to be built on past experience, but must also harness the potential advantages of the raw acceleration signal data. As was the case with counts and regression-based approaches, it will be important to recognise the limitations of raw signal-based analyses, but novel approaches to analyse highresolution data provide the potential to expand the capabilities of these new device technologies and to improve PA research.

\section{PROSPECTS AND ADVANCES IN ACCELEROMETER METHODS}

The potential to model PAEE from counts was recognised at the beginning of the development of modern accelerometers, ${ }^{1}$ and the simplicity of linear regression approaches for developing and applying counts made this approach exceedingly popular with many researchers. Although most of the calibration regression equations estimate average PAEE relatively well for groups (of generally healthy adults and children), the challenges of predicting PAEE accurately for individuals and over a wide range of activities are also well known. ${ }^{5}$ The large errors associated with EE estimates for individuals preclude the use of accelerometers to calibrate dietary intake for energy balance or estimate changes in PAEE in response to an intervention, two applications for which there is high demand. ${ }^{6}$ Moreover, multiple calibration studies have generated widely divergent regression models for converting counts to PAEE, yielding different cutpoints for PA categories. ${ }^{7}$ These diverse equations and cutpoints created considerable confusion and frustration for PA and other health researchers who wished to select the most appropriate way to analyse their accelerometer data. ${ }^{7-9}$

A noteworthy shift in the past decade was the demonstration of significantly improved PAEE estimation compared with regression calibrations by using signal features and patterns extracted from raw acceleration data with machine-learning techniques to derive more sophisticated models. ${ }^{10}$ Through the model development processes, researchers also recognised that PAEE was not the only outcome variable that could be extracted from acceleration signals. With the implementation of piezoresistive and capacitive accelerometer transducers, static acceleration (the direct current or DC component) from the raw signals can be used to estimate limb angles and thus infer postures. ${ }^{11}$ Combining the positional information with the movement acceleration data (the alternating current or AC component) in orthogonal directions provides rich feature sets that allow modelling experts and statisticians to utilise the power of pattern recognition, machine learning and fusion of different techniques to respond to an ever-expanding application field. ${ }^{12}$ The ability to differentiate PA types is providing new insights and promises to expand the scope of PA research in behavioural and clinical sciences.

Accompanying the enthusiasm regarding high-resolution raw acceleration signal capture are concerns related to storage and transmission of the high-data volumes as well as appropriate data modelling methods. With rapidly expanding computer memory sizes at comparable or lower cost, storage is no longer a significant limitation. Data transfer from the onboard memory of raw-data accelerometers (about 0.5 GB for each 7-day collection) can now be performed within minutes. However, it is currently challenging to translate the raw data to the desirable results of PA types and PAEE. The raw-data-based analytic models, particularly multidimensional algorithms, are still being developed, validated and optimised by researchers and device manufacturers. However, the widespread interest in 'big data' provides analytic approaches that are being applied to accelerometer signal data.

To reduce barriers to adoption and support replication and cross-validation of new models, the models need to be built into easy-to-use software or in open-source shareware forms so that they are useful for applied researchers and clinicians. A number of efforts are currently under way within the academic, small business and government sectors to address the specific computational requirements to implement signal processing methods for large volumes (eg, Terabytes) of acceleration and related sensor (eg, gyroscope or heart rate) data. For example, the US National Cancer Institute has supported the development of scalable systems for collection, storage, analysis and reporting of data from diverse sensor platforms via Small Business Innovation Research (SBIR) contracts. A specific requirement of these systems was the implementation of fully transparent (and customisable) analytic tools to process data from raw sensor 
signals into outcome measures. Device manufacturers and application developers have also continued to invest in software solutions or support for open-source tools (eg, such as R-code and libraries) in order to support their users' analytic needs. The availability of efficient raw signal data analytic approaches will ultimately encourage researchers towards new models of accelerometer data analysis. These new models may decrease reliance on batch processing on desktop computers and increase implementation of rolling data analysis, perhaps on cloud-based computing platforms.

Another concern within the PA research field is the comparability and accuracy of information extracted from acceleration signals recorded from different body locations. For example, the correlation between activity counts and PAEE from uniaxial accelerometers was found to be much lower when positioned on the wrist rather than at the hip. ${ }^{13}$ However, several recent studies that used features from triaxial raw accelerometer signals have narrowed the gap between PAEE estimates from wristworn and hip worn-accelerometers ${ }^{14} 15$ and for classifying PA into sedentary, household, walking and running types. ${ }^{16}$ Such efforts will certainly grow and mature over the next few years.

Current accelerometer-based devices have moved beyond small-capacity $(<1 \mathrm{MB})$ onboard memory chips and piezoelectric sensors, which are now expensive and difficult for device manufacturers to find. In the near future, the PA field may also move beyond reliance on count-based linear regressions and cutpoints for data extraction from accelerometers.

\section{COLLABORATION TO STIMULATE METHODOLOGICAL PROGRESS}

As analysis methods are developed for raw acceleration signals, it would be advantageous to PA researchers if methods evolved towards a consensus approach. Such a consensus approach would facilitate comparison of results across multiple and diverse studies. With the movement towards raw accelerometer data capture, it may even be possible to have approaches to allow direct comparison of results from different manufacturers' devices. This result would contrast with the previous development of device-specific cut-point-based approaches for calibration of accelerometer-based devices, where results from different devices could not be directly compared and so many different analysis approaches existed that it was possible to shape the outcome of a study by the choice of a cut-point. Examples of areas where agreement could accelerate progress are: core activities to include in protocols, standardised naming conventions for variables in calibration studies or other data collections, choice of machine learning approach and potential signal features to include in algorithm development.

At the 3rd International Conference on Ambulatory Monitoring of Physical Activity and Movement (ICAMPAM) in June 2013, a preconference workshop was held to propose a collaborative approach to algorithm development. After the conference, an email was sent to all attendees inviting involvement in the collaborative effort. Approximately 90 participants responded, indicating interest in collaboration, with many expressing interest in sharing data to facilitate development of a consensus analytic approach. At this time, smaller working groups are being formed to propose solutions to issues such as ethical and privacy issues, harmonising calibration protocols, harmonising data elements for collaboration, data management logistics, sensor technology and necessary metadata, machine learning and analytic methods, and evaluating evidence for comparison of analysis methods. Efficient models for dissemination of the results of these deliberations will be explored.

\section{ACCELEROMETER DEPLOYMENTS AND PROTOCOLS IN THE US NHANES}

In the 2003-2004 and 2005-2006 cycles of the US NHANES, survey, participants aged 6 years and older were asked to wear an Actigraph 7164 on a waist belt during all non-sleeping hours for 7 days. At the time, this data collection on nearly 15000 individuals was the largest accelerometer deployment ever undertaken. Partially due to the success of accelerometers in NHANES, national studies in Canada, ${ }^{17}{ }^{18}$ England $^{19}$ and Finland (Tommi Vasankari, personal communication, 2013) have deployed accelerometer-based devices and numerous cohort studies have included accelerometer measures. The NHANES accelerometer data have been widely used by researchers to explore relationships among accelerometer measures and a variety of other measures. ${ }^{20}$ Despite the success of using an accelerometer device in NHANES and the widely used data, participant compliance was recognised as a major issue. Only about $25 \%$ of participants provided the requested 7 days of data, which was mostly attributed to the discomfort or inconvenience of wearing a device on the hip over time, and forgetting to put the monitor back on after taking it off at night.

Accelerometer-based devices returned to NHANES in the 2011-2012 and 2013-2014 cycles. However, the device and protocol used differed from the earlier NHANES. The Actigraph was used, but the then-current model (GT3X+) was waterproof and supported collection of 7 days of continuous triaxial accelerometer raw-signal data at $80 \mathrm{~Hz}$. Rather than repeating the waist location used in 2003-2006, wrist wear on the non-dominant wrist was chosen. Wrist wear of a waterproof device would allow the device to be worn around the clock for 7 days. This body location and protocol was chosen to improve wear compliance and had the additional benefit of allowing the measurement of movement during sleep. Wrist actigraphy is a well-validated measure of sleep duration and quality. ${ }^{21}$ However, for PA monitoring, use of the dominant versus nondominant wrist remains an open question. The selection of the non-dominant wrist for the current NHANES protocol was predicated on a historical precedent for sleep research and early reports of wrist-device calibration results, ${ }^{15}$ and should not yet be considered as a standard for $24 \mathrm{~h}$ accelerometer-based PA protocols. At this point, early wrist accelerometer calibration studies do not appear to strongly favour right versus left wrist (or dominant vs non-dominant) for PA monitoring. ${ }^{14-16}$

Preliminary data suggest that the protocol change had the desired effect on compliance. In the 2003-2004 cycle of NHANES, approximately $40-70 \%$ of participants by age group provided at least 6 days of data with at least $10 \mathrm{~h}$ of wear time and a large effect of age on wear compliance was evident. In contrast, in the 2011-2012 cycle with wrist wear, $70-80 \%$ of participants provided at least 6 days of data with at least $18 \mathrm{~h}$ of wear time and little difference by age was apparent. Of participants who provided at least one acceptable wear day, 70\% provided seven such days. The resulting median wear time in 2011-2012 was approximately 22 h/day.

Perhaps influenced by the protocol choice in NHANES, other researchers have included wrist-worn accelerometer devices in their studies. $^{22}$ Many researchers across the fields of PA, machine learning and statistics have also begun to explore approaches to translate raw accelerometer signals from the wrist to activity classification. ${ }^{11} 14-16$ 


\section{COMPARING ACCELEROMETER AND SELF-REPORT DATA: TIME FOR REFLECTION}

The term PA is used to describe various aspects of an individual's daily behaviours (sports, occupation), activities (walking, running, swimming) and bodily movements. Summary measures of PA extracted from motion sensors and self-report instruments have commonly been expressed using nominally the same metrics, such as minutes/day in specific intensity categories (eg, moderate-to-vigorous PA (MVPA) minutes or MET-minutes). As has been repeatedly demonstrated in studies that compare questionnaires and accelerometer outcomes, the measures have a low to moderate correlation, ${ }^{23}{ }^{24}$ but these distinct assessment methods are not equivalent.

Accelerometer-based monitors quantify acceleration resulting from PA-associated bodily motion at a fixed point of the body (ie, hip, wrist, thigh, etc). ${ }^{4}$ Data outputs from accelerometers, such as filtered activity counts or raw acceleration signals, provide an explicit representation of acceleration due to bodily motion over short time periods determined by the device settings, generally fractions of a second to a minute. As a consequence, outputs derived from motion sensor data, such as MVPA minutes, reflect the device resolution of time and detected acceleration.

In contrast, self report instruments attempt to quantify PA based on reported time periods engaged in specific behaviours of longer duration. Behavioural self-report provides an integrated proxy measure for bodily motion that may incorporate elements of psychosocial and environmental context, activity purpose, perceived time-use and intensity of effort. Many behaviours that are specifically queried or reported on PA self-report instruments consist of patterns or groupings of specific activities (eg, sitting, standing, walking, bending, lifting, throwing and kicking) that are non-continuous in nature or activities for which EE may be poorly quantified by a typically waist worn accelerometer (eg, cycling). Either the high-resolution device measure or the longer time scale of reports can be 'correct' within the construct assessed by each instrument (movement vs behaviour). However, the time distinction and different levels of aggregation lead to challenges for direct comparison of outcomes.

An example of the effect of the distinction between the types of measure is apparent in the study by Chastin et al, ${ }^{25}$ which found that among delivery postal workers who walked a route, $77 \%$ met the recommendation of 10000 steps/day on five or more days, but only $3 \%$ met the recommendation of at least 5 days of $30 \mathrm{~min}$ or more per day of moderate intensity activity with a $10 \mathrm{~min}$ bout requirement. These workers would be very likely to report that they walked several hours per day. The authors note that self-reports consider the perceived total duration, but do not account for the actual fragmentation of activity-related movement. In the Chastin et al study, ${ }^{23}$ a pause of $10 \mathrm{~s}$ during walking was sufficient to interrupt a walking bout. This is one example, but there are many other instances where a truthful report of PA behaviour will not translate to a continuous bout of movement of similar duration measured by an accelerometer-based device.

The inclusion of accelerometers in NHANES 2003-2004 and 2005-2006 allowed analyses that compared the prevalence of meeting PA recommendations as measured by accelerometer to the prevalence based on self-reported PA collected within the survey. $^{26} 27$ These analyses found dramatic differences in the prevalence estimates by measurement method. However, evaluation of PA guideline adherence based on accelerometer outcomes is inappropriate because the behavioural metrics used to develop the guidelines differ conceptually from device-based measures of MVPA.

The epidemiological studies that are the basis for establishing the Physical Activity Guidelines for Americans ${ }^{28}$ and earlier PA for health recommendations ${ }^{29-31}$ primarily linked health outcomes to self-reported PA from cohort or other observational studies. The questionnaires used queried behaviours that often occur within specific contexts, such as occupation, household tasks, sports and recreation, or transportation walking or bicycling. Accelerometer-based devices accurately measure the movement associated with PA behaviours that may be reported. However, devices are not simply better measures of the same construct captured by questionnaires, despite the reference to both approaches as measures of PA.

Accelerometer-based devices will increasingly be used to characterise the PA patterns of individuals and groups. Eventually, health-related guidelines based on device-measured movement will be developed, and by that time incorporation of accelerometer-based devices into ubiquitous consumer devices may allow easy tracking of compliance with the recommended amount of movement. This vision will be aided by a consensus approach to accelerometer signal analysis. However, until that time, it is important that PA researchers understand the distinction between PA measured with an accelerometer-based device and PA reported on questionnaires, diaries or logs. Each approach has strengths that may be complementary, but devicebased measures and behavioural reports are not interchangeable. $^{32}$

\section{SUMMARY}

The technology and application of accelerometer-based devices in PA research have experienced tremendous advances and growth. Current hardware technology allows the capture and storage or transmission of large volumes of raw acceleration signal data. These data provide opportunities to improve characterisation of PA, but the opportunities are accompanied by logistical and analytic challenges. Researchers and developers from multiple disciplines are responding to the analytic challenges and advances in data storage, transmission and big data computing will minimise logistical challenges. However, the new approaches bring the need for several paradigm shifts for PA researchers, including a shift from count-based approaches and regression calibrations for PAEE estimation to activity characterisation and EE estimation based on features extracted from raw acceleration signals. Furthermore, a collaborative approach towards a consensus analytic method would facilitate PA research, but requires a shift away from multiple independent calibration studies.

As our recognition of the different characteristics of PA evolves and our interests in different aspects of PA behaviour deepen and expand, the distinction between PA represented by accelerometer-based devices and PA as reported by self-report has to be recognised. PA researchers need to be aware that PA estimates derived from these types of approaches are not conceptually equivalent; they may be expressed in the same metrics, but they are not assessing the same thing. As more measures based on devices are used in PA research studies and reported in the health literature, it will be important to recognise the likely error of making inappropriate direct data comparisons. 


\section{What this review adds?}

- Logistical challenges of storing, transmitting and analysing raw acceleration signal data are being addressed by technology and research advances.

- Raw acceleration signal data inspire new paradigms of movement data interpretation.

- Increased understanding of movement data highlights the distinct measurement features of devices and behavioural reports as well as appropriate and complementary applications of each modality.

Contributors RPT, JJM, RJB and KYC contributed to the conception and design, as well as the drafting and revision of the article. RPT is responsible for the overall content and is the guarantor. All the authors have approved the final version.

Competing interests The manuscript and work described were performed by all authors in their roles as US federal employees.

Provenance and peer review Not commissioned; externally peer reviewed.

\section{REFERENCES}

1 Montoye HJ, Washburn R, Servais $S$, et al. Estimation of energy expenditure by a portable accelerometer. Med Sci Sports Exerc 1983;15:403-7.

2 Chen KY, Bassett DR Jr. The technology of accelerometry-based activity monitors: current and future. Med Sci Sports Exerc 2005;37:S490-500.

3 Troiano RP. A timely meeting: objective measurement of physical activity. Med Sci Sports Exerc 2005;37:S487-9.

4 Chen KY, Janz KF, Zhu W, et al. Redefining the roles of sensors in objective physical activity monitoring. Med Sci Sports Exerc 2012;44:S13-23.

5 Plasqui G, Bonomi AG, Westerterp KR. Daily physical activity assessment with accelerometers: new insights and validation studies. Obes Rev 2013;14:451-62.

6 Welk GJ. Principles of design and analyses for the calibration of accelerometry-based activity monitors. Med Sci Sports Exerc 2005;37:S501-11.

7 Matthews CE. Calibration of accelerometer output for adults. Med Sci Sports Exerc 2005;37:S512-22.

8 Freedson P, Pober D, Janz KF. Calibration of accelerometer output for children. Med Sci Sports Exerc 2005;37:S523-30.

9 Rothney MP, Schaefer EV, Neumann MM, et al. Validity of physical activity intensity predictions by ActiGraph, Actical, and RT3 accelerometers. Obesity (Silver Spring) 2008;16:1946-52.

10 Liu S, Gao RX, Freedson PS. Computational methods for estimating energy expenditure in human physical activities. Med Sci Sports Exerc 2012;44:2138-46.

11 Rowlands AV, Olds TS, Hillsdon M, et al. Assessing Sedentary Behavior with the GENEActiv: Introducing the Sedentary Sphere. Med Sci Sports Exerc 2013. [Epub ahead of print]. doi:10.1249/MSS.0000000000000224

12 Intille SS, Lester J, Sallis JF, et al. New horizons in sensor development. Med Sci Sports Exerc 2012;44:S24-31.
13 Swartz AM, Strath SJ, Bassett DR Jr, et al. Estimation of energy expenditure using CSA accelerometers at hip and wrist sites. Med Sci Sports Exerc 2000;32: S450-6.

14 Phillips LR, Parfitt G, Rowlands AV. Calibration of the GENEA accelerometer for assessment of physical activity intensity in children. I Sci Med Sport 2013;16:124-8.

15 Esliger DW, Rowlands AV, Hurst TL, et al. Validation of the GENEA accelerometer. Med Sci Sports Exerc 2011;43:1085-93.

16 Zhang S, Rowlands AV, Murray $P$, et al. Physical activity classification using the GENEA wrist-worn accelerometer. Med Sci Sports Exerc 2012;44:742-8.

17 Colley RC, Garriguet D, Janssen I, et al. Physical activity of Canadian adults: accelerometer results from the 2007 to 2009 Canadian Health Measures Survey. Health Rep 2011;22:7-14.

18 Colley RC, Garriguet D, Janssen I, et al. Physical activity of Canadian children and youth: accelerometer results from the 2007 to 2009 Canadian Health Measures Survey. Health Rep 2011;22:15-23.

19 O'Donovan G, Hillsdon M, Ukoumunne OC, et al. Objectively measured physical activity, cardiorespiratory fitness and cardiometabolic risk factors in the Health Survey for England. Prev Med 2013;57:201-5.

20 Tudor-Locke C, Camhi SM, Troiano RP. A catalog of rules, variables, and definitions applied to accelerometer data in the National Health and Nutrition Examination Survey, 2003-2006. Prev Chronic Dis 2012;9:E113.

21 Morgenthaler T, Alessi C, Friedman L, et al. Practice parameters for the use of actigraphy in the assessment of sleep and sleep disorders: an update for 2007; Standards of Practice Committee; American Academy of Sleep Medicine. Sleep 2007;30:519-29

22 Knuth AG, Assunção MC, Gonçalves H, et al. [Methodological description of accelerometry for measuring physical activity in the 1993 and 2004 Pelotas (Brazil) birth cohorts] [Article in Portuguese]. Cad Saude Publica 2013;29:557-65.

23 Sallis JF, Saelens BE. Assessment of physical activity by self-report: status limitations, and future directions. Res Q Exerc Sport 2000;71:1-14.

24 Dyrstad SM, Hansen BH, Holme IM, et al. Comparison of self-reported versus accelerometer-measured physical activity. Med Sci Sports Exerc 2014;46:99-106.

25 Chastin SFM, Dall PM, Tigbe WW, et al. Compliance with physical activity guidelines in a group of UK-based postal workers using an objective monitoring technique. Eur J Appl Physiol 2009;106:893-9.

26 Troiano RP, Berrigan D, Dodd KW, et al. Physical activity in the United States measured by accelerometer. Med Sci Sports Exerc 2008;40:181-8.

27 Tucker JM, Welk GJ, Beyler NK. Physical activity in US adults: compliance with the Physical Activity Guidelines for Americans. Am J Prev Med 2011;40:454-61.

28 Physical Activity Guidelines Advisory Committee. Physical activity guidelines advisory committee report, 2008. Washington, DC: US Department of Health and Human Services, 2008:683.

29 Pate RR, Pratt M, Blair SN, et al. Physical activity and public health. A recommendation from the Centers for Disease Control and Prevention and the American College of Sports Medicine. JAMA 1995;273:402-7.

30 Haskell WL, Lee IM, Pate RR, et al. Physical activity and public health: updated recommendation for adults from the American College of Sports Medicine and the American Heart Association. Circulation 2007;116:1081-93.

31 Nelson ME, Rejeski WJ, Blair SN, et al. Physical activity and public health in older adults: recommendation from the American College of Sports Medicine and the American Heart Association. Med Sci Sports Exerc 2007;39:1435-45.

32 Troiano RP, Pettee Gabriel KK, Welk GJ, et al. Reported physical activity and sedentary behavior: why do you ask? J Phys Act Health 2012;9:568-75. 